\title{
Global population structure of the stable fly (Stomoxys calcitrans) inferred by mitochondrial and nuclear sequence data
}

\author{
N. Dsouli-Aymes ${ }^{\mathrm{a}, *}$, J. Michaux ${ }^{\mathrm{b}}$, E. De Stordeur ${ }^{\mathrm{a}}$, A. Couloux ${ }^{\mathrm{c}}$, M. Veuille $^{\mathrm{d}}$, G. Duvallet $^{\mathrm{a}}$ \\ a Centre d'Ecologie fonctionnelle et évolutive UMR 5175 CEFE, Université Montpellier 3, Route de Mende, 34199 Montpellier Cedex 5, France \\ ${ }^{\mathrm{b}}$ Centre de Biologie et de Gestion des Populations, Montpellier, France \\ ${ }^{\mathrm{c}}$ Centre National de Séquençage, Evry Cedex, France \\ ${ }^{\mathrm{d}}$ Biologie Intégrative des Populations, Muséum National d'Histoire Naturelle, Paris, France
}

\section{A R T I C L E I N F O}

\section{Article history:}

Received 7 May 2010

Received in revised form 3 November 2010

Accepted 5 November 2010

Available online 18 November 2010

\section{Keywords:}

Stomoxys calcitrans

Mitochondrial and nuclear DNA

Phylogeography

Genetic structure

Population expansion

\begin{abstract}
A B S T R A C T
Stomoxys calcitrans (Diptera: Muscidae: Stomoxyini), a synanthropic fly with a worldwide distribution, is recognized to have an important medical and veterinary impact. We conducted a phylogeographic analysis based on several populations from five major zoogeographic regions of the world in order to analyse population genetic structure of $S$. calcitrans and to trace its global dispersion. Results from mitochondrial (COI, Cyt-b and ND1-16S) and nuclear (ITS2) DNA show a substantial differentiation of Oriental populations (first lineage) from the Afrotropical, Palearctic, Nearctic, Neotropical and Oceanian populations (second lineage). The divergence time analyses suggest the separation between the two lineages approximately in mid-Pleistocene. Oriental populations are isolated and would not have participated in the colonization of other regions, unlike the Afrotropical one which seems to be the source of $S$. calcitrans dispersion towards other regions. Demographic analyses indicate that Oriental, Afrotropical and Palearctic regions have undergone a population expansion during late Pleistocene-early Holocene. The expansion time of this cosmopolitan species could have been influenced by continental human expansions and by animal domestication.
\end{abstract}

(c) 2010 Elsevier B.V. All rights reserved.

\section{Introduction}

Stomoxys calcitrans (Diptera: Muscidae: Stomoxyini), commonly called stable fly, belongs to the genus Stomoxys Geoffroy, which includes 18 recognized species (Zumpt, 1973). Among these species, only $S$. calcitrans has a worldwide distribution and is a synanthropic fly. All other Stomoxys species are exclusively tropical, twelve of which are located on the African continent, four on the Asian continent, and one species, S. sitiens Rondani, has been reported in both Africa and Asia (Zumpt, 1973). Stable fly is recognized to have an important medical and veterinary impact. Both sexes are hematophagous and make numerous visits to their hosts, bit repeatedly before obtaining a full meal. Economic losses in the cattle breeding caused by stables flies are mainly associated with their direct pathogenic effects: a loss of weight, milk production and immunizing defences (Bruce and Decker, 1958; Miller et al., 1973; Campbell et al., 1977, 2001; Wieman et al., 1992 ) and also by their role in the transmission of virus, bacteria, protozoa and helminths (Zumpt, 1973; Foil et al., 1983; Freitas and Romero, 1991; D'Amico et al., 1996; Foil and Gorham, 2000). Stable

\footnotetext{
* Corresponding author. Tel.: +33 0467142315

E-mail address: dsouli2005@yahoo.fr (N. Dsouli-Aymes).
}

flies are found essentially in rural areas near stables, slaughter houses, cattle markets and the rubbish dumps, locations related mainly to the presence of fermenting organic material (Batista et al., 2005; Eesa and El-Sibae, 1993).

The stable fly is a multivoltine species; the generation time over the year is principally dependent on temperature. Extreme temperatures appear to reduce the duration of egg production (Lysyk, 1998). Ecological evidence suggests that stable flies are strongly vagile, dispersing far and wide (Eddy et al., 1962). These flies have great capacities of flight and certain studies (Bailey et al., 1979; Hogsette et al., 1987) mention surprising displacement distances (several hundred kilometers), in search of a blood meal. Hogsette et al. (1987) showed that $S$. calcitrans can cross $5 \mathrm{~km}$ or more in order to find a blood meal. Marked flies were found $100 \mathrm{~km}$ far of their marking place and even up to $225 \mathrm{~km}$ after a storm (Foil and Hogsette, 1994).

Recently, Marquez et al. (2007) published the first and the unique valuable work on stable fly phylogeography using mitochondrial DNA COI (550 bp) and r16S DNA (300 bp). They explained the geographic origin of New World $S$. calcitrans populations, and concluded that the most parsimonious scenario is that New World Stomoxys flies had Palearctic origins within the past 500 years.

In the present study we examine the population structure and demographic history of $S$. calcitrans collected from different 
zoogeographic regions using mitochondrial DNA (COI, Cyt-b and ND1-16S) and nuclear DNA (internal transcribed spacer regions of the 18S-26S ribosomal RNA genes, ITS2) sequences.

\section{Materials and methods}

The specimens used in this study were provided by numerous colleagues and were collected from twenty countries from different zoogeographic regions. Vavoua traps (Laveissière and Grébaut, 1990) were used for capture. Trapped flies were put into freezer to kill them, and then they were preserved at room temperature in $95 \%$ ethanol and identified in the laboratory according to Zumpt (1973). Specimens are available upon request from the corresponding author and are stored at the Laboratoire de Zoogéographie, Université Paul-Valéry, Montpellier (France).

\subsection{DNA extraction, DNA amplification and sequencing}

Genomic DNA was extracted using the DNeasy tissue Kit (QIAGEN) to a final volume of $180 \mu \mathrm{l}$. Primer sequences for mtDNA were defined according to Simon et al. (1994, 2006): TL2-N-3014 (TCCATTGCACTAATCTGCCATATTA) and C1-J-2183 (CAACATTTATTTTGATTTTTTGG) for COI; CB-J10933 (GTTTTACCTTGAGGACAAATATC) and CB-N11526 (TTCAACTGGTCGAGCTCCAATTCA) for Cyt-b; N1-N12261 (AACTTCATAAGAAATAGTTTGAGC) and LRN13000 (TTACTTTAGGGATAACAGCGTAA) for ND1-16S. Primers sequences for ITS2 were defined according to Sharpe et al. (2000): ITS2A (TGTGAACTGCAGGACACAT) and ITS2B (TATGCTTAAATTCAGGGGGT). Standard protocols were used for all PCR amplifications. They were performed in a $30 \mu \mathrm{l}$ reaction volume containing, in final concentrations, $200 \mu \mathrm{M}$ dNTPs (diNucleotide Tri Phosphate), $10 \times$ buffers, $25 \mu \mathrm{M}$ of each primers, $0.5 \mu$ l Taq polymerase (Eurogentec Red GoldStar ${ }^{\circledR}$ ), and $3 \mu$ l of $180 \mu$ l total volume of DNA solution extraction. Thermal cycling conditions were initial denaturation at $94{ }^{\circ} \mathrm{C}$ for $4 \mathrm{~min}, 35$ cycles of denaturation at $94{ }^{\circ} \mathrm{C}$ for $40 \mathrm{~s}$, annealing at $48-50{ }^{\circ} \mathrm{C}$ for COI, $57-58{ }^{\circ} \mathrm{C}$ for Cyt-b, $53-54{ }^{\circ} \mathrm{C}$ for ND1-16S and $60-62{ }^{\circ} \mathrm{C}$ for ITS2, and extension at $72{ }^{\circ} \mathrm{C}$ for $1 \mathrm{~min}$. An elongation step at $72{ }^{\circ} \mathrm{C}$ for $10 \mathrm{~min}$ completed the DNA amplification. One to ten specimens for each $S$. calcitrans populations were sequenced (Table 1 ), using Sanger sequencing on an ABI 3730 automatic sequencer at the Centre National de

Table 1

Number of $S$. calcitrans sequences used for each genes and sampling locations.

\begin{tabular}{llcccc}
\hline Region & Country & COI & Cyt-b & ND1-16S & ITS2 \\
\hline Oriental & 1-India & 2 & 2 & 2 & 1 \\
& 2-Thailand & 9 & 9 & 11 & 12 \\
Afrotropical & 3-Ethiopia & 6 & 4 & 6 & 3 \\
& 4-Uganda & 1 & 2 & 2 & 2 \\
& 5-Gabon & 8 & 6 & 5 & 3 \\
& 6-Cameroun & 3 & 8 & 4 & 9 \\
& 7-Senegal & 6 & 2 & 6 & 5 \\
& 8-Burkina Faso & 1 & 3 & 1 & 0 \\
& 9-Madagascar & 2 & 2 & 1 & 2 \\
Palearctic & 10-La Reunion & 2 & 1 & 2 & 4 \\
& 11-Anjouan & 5 & 5 & 5 & 4 \\
& 12-Mayotte & 1 & 1 & 1 & 1 \\
& 13-Tunisia & 3 & 5 & 5 & 3 \\
14-Algeria & 3 & 4 & 3 & 3 \\
Total & 15-Morocco & 6 & 4 & 5 & 3 \\
Nearctic & 16-France & 5 & 6 & 9 & 3 \\
17-Montenegro & 1 & 1 & 1 & 1 \\
& 18-Canada & 7 & 4 & 11 & 7 \\
& 19-Colombia & 6 & 7 & 7 & 5 \\
& 20-N. Caledonia & 4 & 5 & 4 & 4 \\
& 20 pop & 80 & 81 & 91 & 75 \\
\hline
\end{tabular}

Séquençage, Génoscope, Evry (France). Sequences from ITS2, COI, Cyt-b and ND1-16S were aligned using the multiple alignment program ClustalW 1.4 (Thompson et al., 1994) implemented in BioEdit (Hall, 1999). Sequence alignment was adjusted by visual inspection, taking sequences of $S$. calcitrans available in GenBank as a reference for each gene portion. Sequences obtained during the study are submitted to GenBank under the following accession numbers: HM185405-HM185478 for ITS2, HM219010-HM219090 for Cyt-b, HM219091-HM219170 for COI, and HM245660HM245750 for ND1-16S.

\subsection{Data analysis}

Our analysis was based on separate DNA markers since we failed to sequence the same individuals from different genes employed in this study; therefore in this case combining genes decrease the number sequences to examine.

Evaluation of haplotype number and estimation of haplotype diversity $(h)$, as well as nucleotide diversity $(\pi)$ associated with their respective standard divergence (SD), were performed using DNAsp v 4.10 .9 (Rozas et al., 2003). The pattern of haplotype diversity and nucleotide diversity combinations are, according to Avise (2000), indicators of population demographic events.

The model of DNA substitution that best fitted the data was selected by comparing log-likelihood scores of 56 models of base substitutions using the Akaike Information Criterion (AIC) in ModelTest (Posada and Crandall, 1998) using PAUP4.0b10 (Swofford, 2002).

Phylogenetic trees were constructed by maximum likelihood (ML) within PHYML v 2.1b1 (Guindon and Gascuel, 2003), based on the selected model. Nodal support was evaluated by 1000 bootstrap replicates. Musca domestica (Diptera: Muscidae: Muscini) and Haematobia irritans (Diptera: Muscidae: Stomoxyini) were selected as outgroups, for phylogenetic construction. Calculations of genetic distance among sequences were carried out with MEGA4 (Tamura et al., 2004, 2007), according to the best models of the nucleotide substitution.

We also conducted a minimum spanning network among haplotypes from the matrix of pairwise distances calculated among all pairs of haplotypes for each gene portion, using Arlequin v 3.0 (Excoffier et al., 2005).

To assess population's subdivision between zoogeographic regions, we performed an analysis of molecular variance (AMOVA), available in Arlequin v 3.0, using mitochondrial genes.

Inferring the historical demography was conducted by COI, CytB and ND1-16S, for only the Oriental, Afrotropical and the Palearctic populations due to a weak sampling size of remaining populations. Signatures of population demographic changes in $S$. calcitrans were first examined by Tajima's $D(D)$ (Tajima, 1989) and Fu's Fs (Fs) (Fu, 1997) statistics, using Arlequin 3.0, to see whether $S$. calcitrans data conformed to expectations of neutrality or departed from neutrality due to factors such as a population bottleneck or expansion. The examination of deviation from neutrality by both $D$ and $F$ s indices was based on 1000 coalescent simulations. Expectations of these statistics are nearly zero in a constant-size population; significant negative values signify a sudden expansion in population size, whereas significant positive values indicate processes such as a population subdivision or recent population bottleneck. Second demographic changes in $S$. calcitrans were also investigated by comparing mismatch distributions in each geographic sample with those expected in stationary and expanding populations using DnaSP v 4.10.9.

The overall validity of the estimated demographic model was tested by obtaining the distribution of a test statistic SSD (the sum of squared differences) between observed and expected mismatch distributions. A significant SSD value is considered as the evidence 
of departure from the estimated demographic model of a sudden population expansion using Arlequin 3.0. Furthermore, we calculated the raggedness index of the observed mismatch distribution for each of populations according to the population expansion model implemented in Arlequin 3.0. Small raggedness values represent a population which experienced sudden expansion whereas higher values of the raggedness index suggest stationary or bottleneck populations.

Finally, if distribution fits the expansion model, then the time elapsed since the demographic event can be estimated by the parameter Tau $(\tau)$, which is the date of the demographic events measured in the units of mutational time (Rogers and Harpending, 1992). Subsequently, we estimated the time since the most recent expansion $(t)$ from the mismatch distribution parameter $\tau$ using the equation $\tau=2 u t$ (Rogers and Harpending, 1992), where $u$ is the mutation rate per sequence and per generation $\left(u=m_{T} \mu\right.$ where $m_{T}$ is the number of nucleotides of the sequences under study and $\mu$ is the mutation rate per nucleotide). A calibrated molecular clock is not available for Stomoxys, thus we have applied the commonly used standard rate of $2.3 \%$ pairwise sequence divergence per million years for mitochondrial DNA in arthropods (Brower, 1994).

\section{Results}

\subsection{ITS2 analysis}

Twenty base pairs of the $357 \mathrm{bp}$ ITS2 sequences were polymorphic, containing 11 of parsimony informative sites. The number of ITS2 ribotypes was 25 on 75 sequences. Only one was shared between all zoogeographic regions, except for the Oriental area (Table 2). All other ribotypes were unique to their geographic locations. No deep phylogenetic relationships were resolved in the maximum likelihood tree (Fig. 1a). The Oriental populations (India and Thailand) were separated from the other populations with a weak support (Bootstrap value lower than 50\%). The best model for ITS2 ribotypes was $F 81+\Gamma$. The gamma distribution shape parameter $(\alpha)$ was estimated to 0.360 . ITS2 appeared not to be suitable for evaluating phylogenetic relationships within $S$. calcitrans populations. Network analysis shows few mutational steps and displays a star like pattern, where the most common ribotypes lie at the star's centre and derivatives are connected to them independently by short branches. Overall, ITS2 haplotype and nucleotide diversity were low $(h=0.726 \pm 0.057$ and
$P=0.00493 \pm 0.0007)$. Tajima's $D$ and Fu's $F$ statistics over all samples were significantly negative, with $-1.911(P<0.05)$ and -22.590 $( \pm 0.000)$, respectively.

\subsection{Mitochondrial analysis}

For COI, Cyt-b and ND1-16S, 753 bp, 526 bp and 568 bp, respectively, were analyzed from 20 localities spanning different zoogeographic regions.

Genetic diversity indices for S. calcitrans are shown in Table 3. Nucleotide diversity $(\pi)$ calculated for the different zoogeographical regions for separate mitochondrial markers was low, ranging from 0.00032 to 0.00527 . Haplotype diversity $(h)$ was low to moderate for the major zoogeographical regions, except for the Oriental and the Afrotropical regions, for Cyt-b and ND1-16S, respectively. According to Avise (2000), such low $h$ and $\pi$ values would reveal that major populations underwent recent bottlenecks.

Distribution of shared haplotypes is listed in Table 2. The Oriental region does not share any haplotypes with the other regions. Majority of the New World haplotypes are common either with Palearctic or Afrotropical regions. It is interesting to note that mtDNA data did not record any common haplotypes between Palearctic and Afrotropical regions.

Maximum likelihood analyses of mitochondrial $S$. calcitrans DNA haplotypes provided GTR $+\Gamma$ as the best model of DNA substitution for each gene. Phylogenetic tree reconstructions, conducted by COI, Cyt-B and ND1-16S, illustrate similar topologies characterized by the presence of two clades. The first one is consisting of populations from India and Thailand and the second one includes all the remaining populations from five zoogeographic regions. ML method exhibits a great bootstrap value $(B P=100)$, all over the three genes, separating Oriental from the remaining haplotypes (Fig. 1b-d).

The minimum spanning network for COI, Cyt-b and ND1-16S datasets used separately, confirm that the Oriental populations (group I) was clearly differentiated from the remainders (group II) by 13, 6 and 9 mutational steps for COI, Cyt-b and ND1-16S, respectively (Figs. 2-4).

For the separate mitochondrial DNA datasets, Modeltest found the HKY85 (Hasegawa et al., 1985) to be the best nucleotide substitution model to describe each datasets. As this model is not available for analysis in MEGA or Arlequin, all the analyses were

Table 2

Distribution of shared haplotypes of S. calcitrans among zoogeographic regions.

\begin{tabular}{|c|c|c|c|c|c|c|c|c|}
\hline & Haplotypes no & Afrotropical & Palearctic & Oriental & Nearctic & Neotropical & Oceania & \\
\hline \multirow[t]{6}{*}{$\mathrm{COI}$} & Hap_COI1 & & 6 & & 1 & & & \\
\hline & Hap_COI2 & & 7 & & & 3 & & \\
\hline & Hap_COI4 & 11 & & & 1 & & 1 & \\
\hline & Hap_COI9 & 1 & & & 1 & 3 & & \\
\hline & Prop. singleton & 0.77 & 0.71 & 1 & 0.4 & $0 / 2$ & 0.66 & \\
\hline & Total Hap/N & $9 / 34$ & $7 / 18$ & $6 / 11$ & $5 / 7$ & $2 / 6$ & $3 / 4$ & $26 / 80$ \\
\hline \multirow[t]{6}{*}{ Cyt-b } & Hap_Cytb1 & & 14 & & 1 & 3 & 1 & \\
\hline & Hap_Cytb2 & & 3 & & & 2 & & \\
\hline & Hap_Cytb3 & 13 & & & & 2 & & \\
\hline & Hap_Cytb9 & & & & 1 & & 2 & \\
\hline & Prop. singleton & 0.8 & 0.6 & 1 & 0.33 & 0 & 0.33 & \\
\hline & Total Hap/N & $10 / 34$ & $5 / 20$ & $7 / 11$ & $3 / 4$ & $3 / 7$ & $3 / 5$ & $24 / 81$ \\
\hline \multirow[t]{3}{*}{ ND1-16S } & Hap_ND1-16S1 & & 18 & & 10 & 5 & 2 & \\
\hline & Prop. singleton & 1 & 0.8 & 1 & 0.5 & 0.5 & 0.5 & \\
\hline & Total Hap/N & $17 / 33$ & $5 / 23$ & $4 / 13$ & $2 / 11$ & $2 / 7$ & $2 / 4$ & $29 / 91$ \\
\hline \multirow[t]{3}{*}{ ITS2 } & Rib_ITS2-2 & 17 & 8 & & 5 & 5 & 4 & \\
\hline & Prop. singleton & 0.91 & 0.83 & 1 & 0.5 & 0 & 0 & \\
\hline & Total Rib/N & $12 / 33$ & $6 / 13$ & $6 / 13$ & $2 / 7$ & $1 / 5$ & $1 / 4$ & $25 / 75$ \\
\hline
\end{tabular}

$N$ : number of sequences. 
a

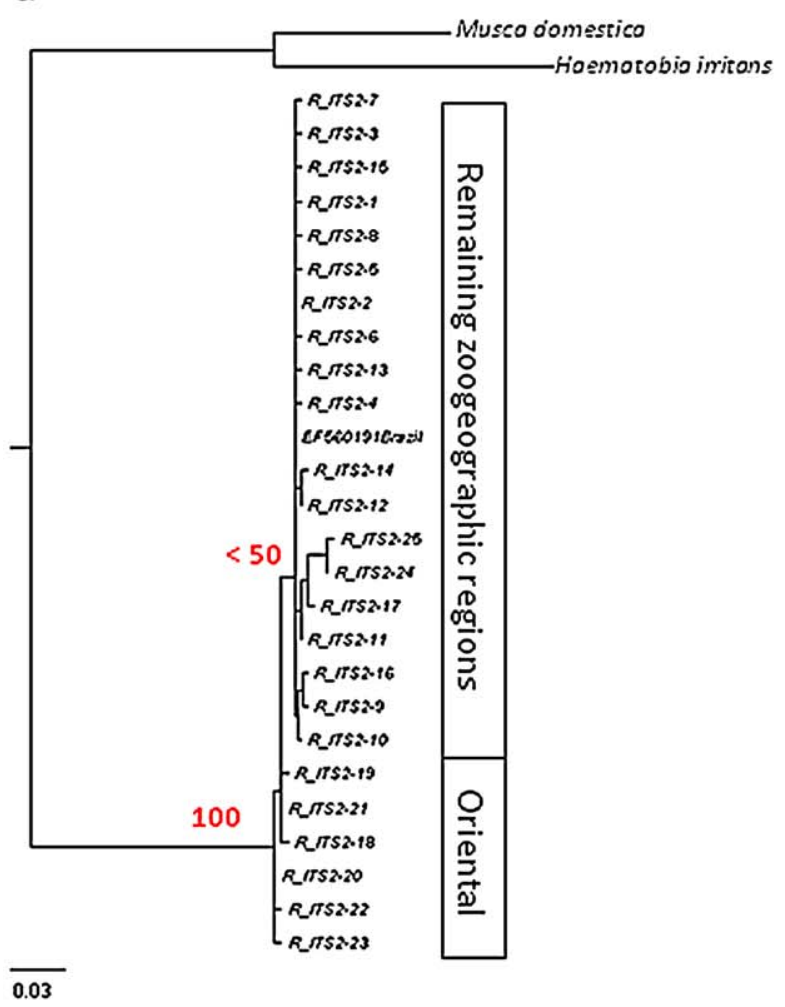

b

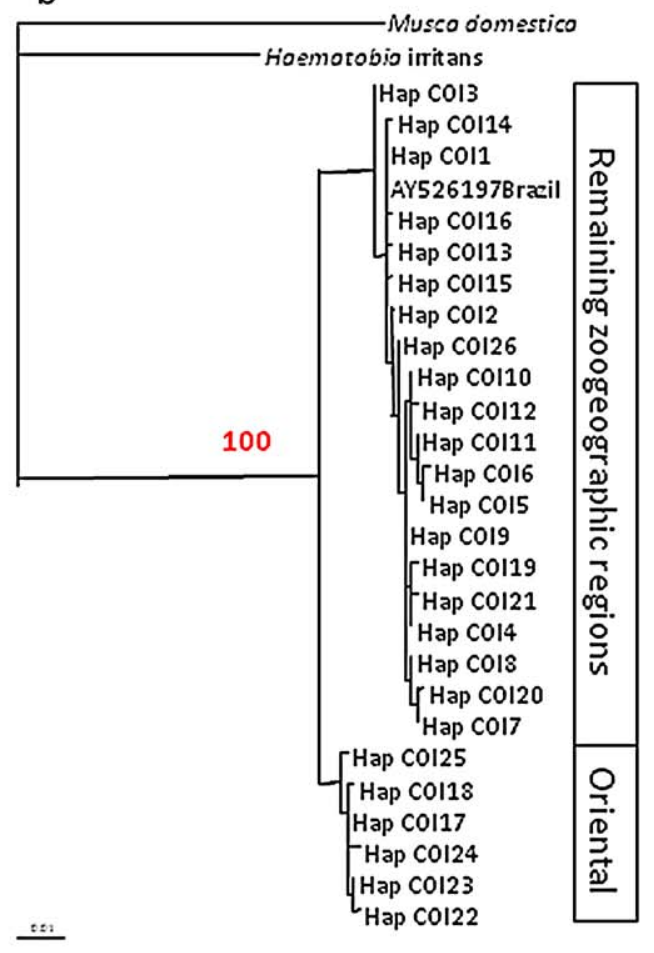

C

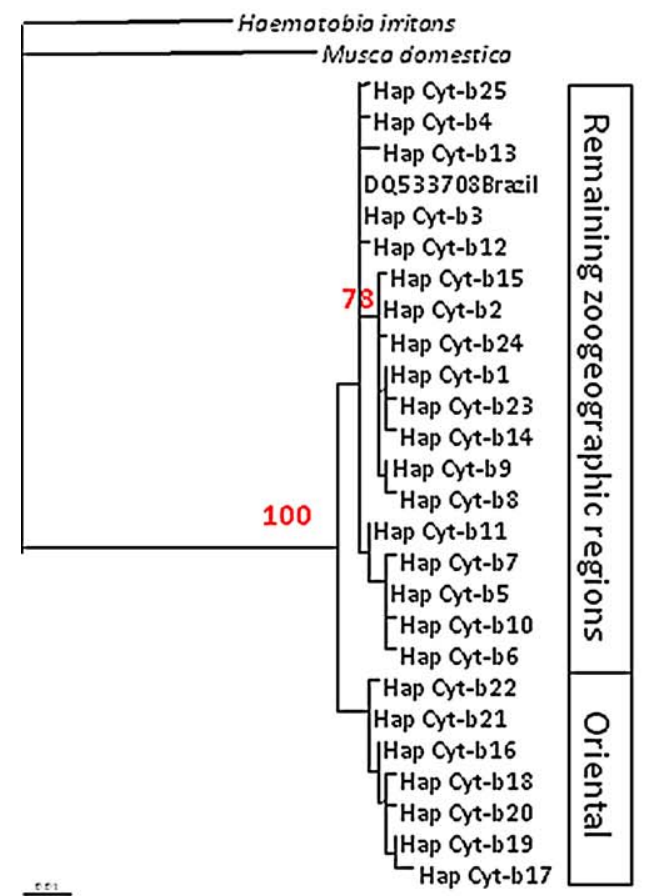

d

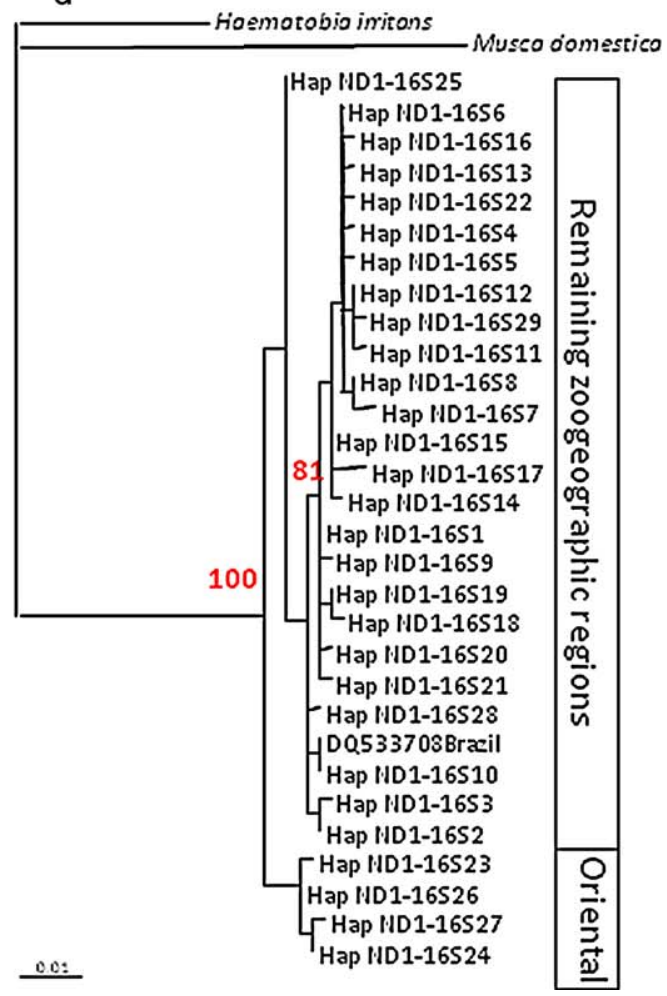

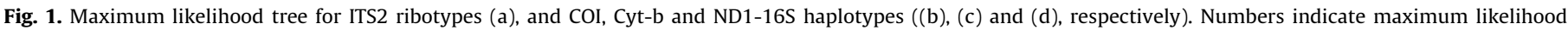
bootstrap proportions.

run using the Tamura and Nei (1993) substitution model that is the most similar to the HKY.

The hierarchical AMOVA conducted among the groups I and II revealed significant structure (Table 4 ), with the main genetic variation attributed to among groups (85.14\% for COI, $71.73 \%$ for Cyt-B and $77.06 \%$ for ND1-16S). Mean genetic distance among sequences of groups I and II was $2.3 \%, 1.9 \%$ and $1.7 \%$ for COI, Cyt-b and ND1-16S, respectively. 
Table 3

Summary data for genetic diversity indices for the different DNA markers in the $S$. calcitrans poulations.

\begin{tabular}{|c|c|c|c|c|c|}
\hline & & $N$ & hap & $h(\mathrm{SD})$ & $\pi(\mathrm{SD})$ \\
\hline \multirow[t]{7}{*}{$\mathrm{COI}$} & Oriental & 11 & 6 & $0.855(0.085)$ & $0.00237(0.00046)$ \\
\hline & Afrotropical & 34 & 9 & $0.847(0.040)$ & $0.00226(0.00022)$ \\
\hline & Palearctic & 18 & 7 & $0.765(0.073)$ & $0.00143(0.00025)$ \\
\hline & Nearctic & 7 & 5 & $0.857(0.198)$ & $0.00215(0.00059)$ \\
\hline & Neotropic & 6 & 2 & $0.600(0.283)$ & $0.00159(0.00034)$ \\
\hline & Oceania & 4 & 3 & $0.833(0.222)$ & $0.00243(0.0007)$ \\
\hline & Total & 80 & 26 & $0.938(0.012)$ & $0.00782(0.00106)$ \\
\hline \multirow[t]{7}{*}{ Cytb } & Oriental & 11 & 7 & $0.909(0.066)$ & $0.00415(0.00059)$ \\
\hline & Afrotropical & 34 & 10 & $0.722(0.054)$ & $0.00383(0.00032)$ \\
\hline & Palearctic & 20 & 5 & $0.507(0.126)$ & $0.00105(0.00030)$ \\
\hline & Nearctic & 4 & 3 & $0.833(0.222)$ & $0.00317(0.00011)$ \\
\hline & Neotropic & 7 & 3 & $0.762(0.115)$ & $0.00290(0.00067)$ \\
\hline & Oceania & 5 & 3 & $0.800(0.164)$ & $0.00304(0.00062)$ \\
\hline & Total & 81 & 24 & $0.885(0.020)$ & $0.00816(0.00080)$ \\
\hline \multirow[t]{7}{*}{ ND1-16S } & Oriental & 13 & 4 & $0.615(0.136)$ & $0.00145(0.00043)$ \\
\hline & Afrotropical & 33 & 17 & $0.933(0.022)$ & $0.00527(0.00042)$ \\
\hline & Palearctic & 23 & 5 & $0.391(0.125)$ & $0.00102(0.00037)$ \\
\hline & Nearctic & 11 & 2 & $0.182(0.144)$ & $0.00032(0.00026)$ \\
\hline & Neotropic & 7 & 2 & $0.476(0.171$ & $0.00168(0.00060)$ \\
\hline & Oceania & 4 & 2 & $0.667(0.204)$ & $0.00352(0.00108)$ \\
\hline & Total & 91 & 29 & $0.885(0.036)$ & $0.00717(0.00072)$ \\
\hline \multirow[t]{7}{*}{ ITS2 } & Oriental & 13 & 6 & $0.846(0.070)$ & $0.00381(0.00049)$ \\
\hline & Afrotropical & 33 & 12 & $0.727(0.081)$ & $0.00430(0.00112)$ \\
\hline & Palearctic & 13 & 6 & $0.641(0.150)$ & $0.00251(0.00079)$ \\
\hline & Nearctic & 7 & 3 & $0.524(0.144)$ & $0.00160(0.00072)$ \\
\hline & Neotropic & 5 & 1 & - & - \\
\hline & Oceania & 4 & 1 & - & - \\
\hline & Total & 75 & 25 & $0.726(0.057)$ & $0.00493(0.00070)$ \\
\hline
\end{tabular}

$N$ : number of sequences; hap: number of haplotype; $h$ : haplotype diversity; $\pi$ : nucleotide diversity.

\subsection{Demographic history}

Neutrality tests of Tajima's $D$ revealed non-significant negatives values in all populations. Non-significant negative Fu's Fs values were also obtained in four cases in the Afrotropical populations detected by COI and Cyt-b separately and in the Oriental populations by COI and ND1-16S separately (Table 5). However, significant negative Fu's $F$ s values $(P<0.02)$ were found for the Palearctic populations with the three mitochondrial genes, for the Oriental population with Cyt-b and for the Afrotropical populations with ND1-16S data (Table 5). The mismatch distributions were unimodal in most cases of the studied populations, at the exception of the Afrotropical ones where the shape was clearly bimodal for the Cytb and ND1-16S (Fig. 5). The mismatch population test statistics SSD and $\mathrm{Rg}$ were small and not statistically significant, indicating that the sudden expansion model could not be rejected.

Time since the most recent expansion was estimated with COI, as the mismatch distributions were unimodal for all considered populations in the demographic analysis. The $\tau$ values obtained were $1.835,2.027$ and 1.263 for Afrotropical, Oriental and Palearctic populations, respectively.

We assume 10 generations per year of $S$. calcitrans in converting the time to expansion to years. The result suggests a range expansion about 10,595 years ago for the Afrotropical populations, about 11,703 years ago for the Oriental populations and 7292 years ago for the Palearctic populations. All of these expansions seem to have occurred during late Pleistocene-early Holocene.

\section{Discussion}

Our investigation on $S$. calcitrans is complementary to that carried out by Marquez et al. (2007) and is based on additional data sets markers. However, by adding the ITS2 ribosomal DNA, we were unable to resolve a deep phylogenetic relationship among $S$. calcitrans populations. Network of ITS2 S. calcitrans was star-like with dominant shared ribotype, which could indicate signature of population expansion. Conversely, phylogenetic and networks analyses based on mtDNA revealed that $S$. calcitrans populations partitioned into two clades, separated by mean genetic distances

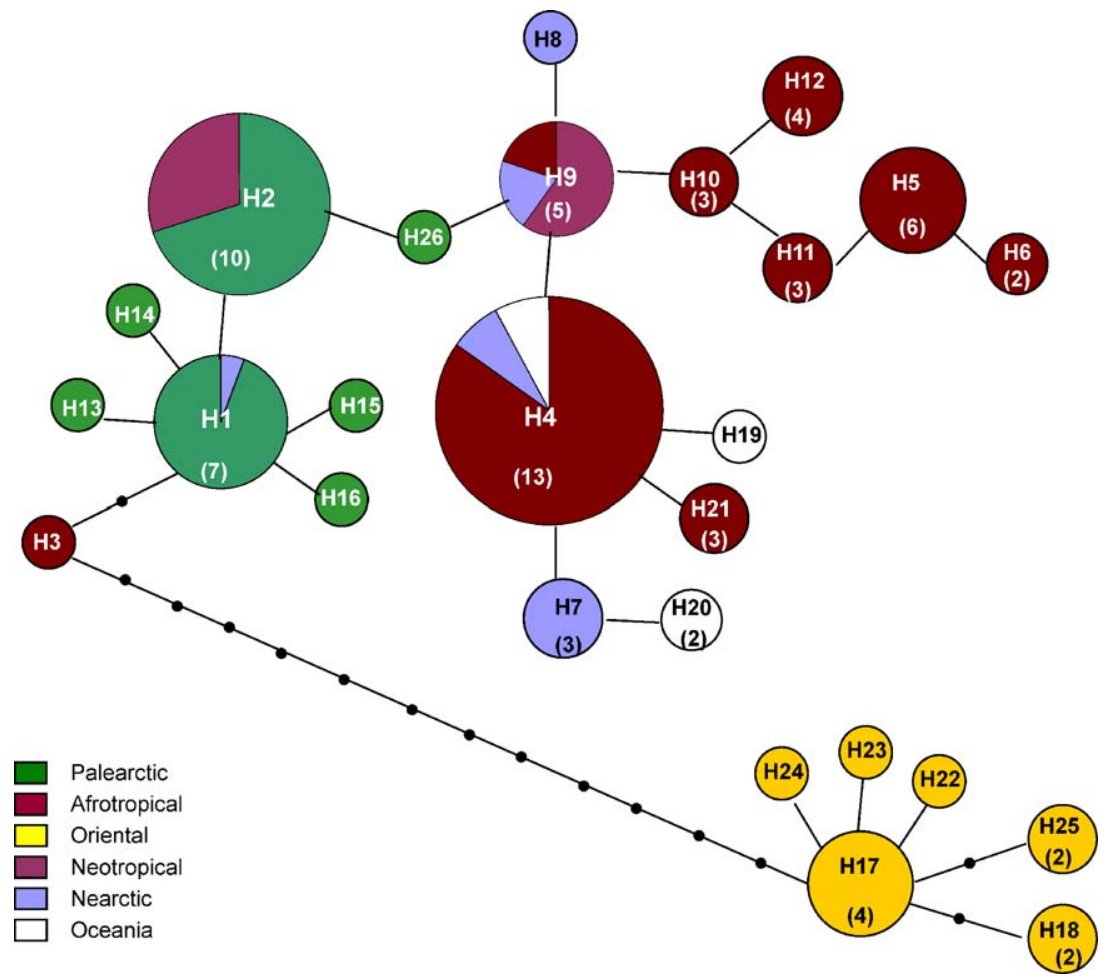

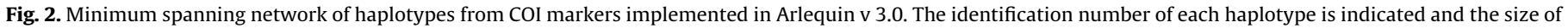
circles is proportional to the number of sequences $(N)$ indicated in brackets $(N=1$ if not otherwise specified). Lines and full circles indicate mutational steps. 


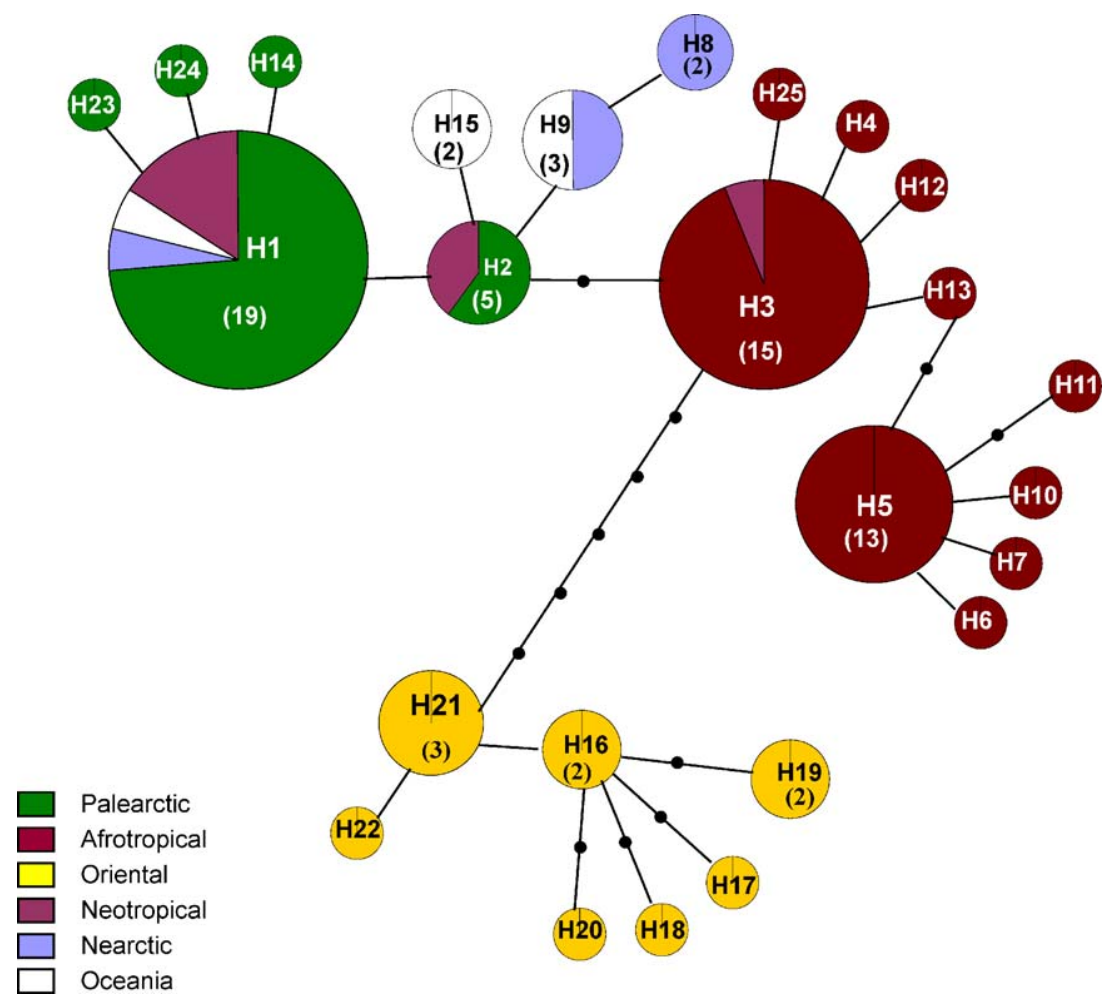

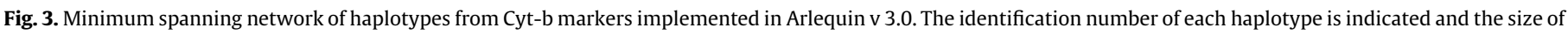
circles is proportional to the number of sequences $(N)$ indicated in brackets $(N=1$ if not otherwise specified). Lines and full circles indicate mutational steps.

(d) of about 1.7-2.3\%. The genetic distance between the two geographically isolated clades, together with the absence of haplotype shared between them, suggest that they represent two distinct evolutionary lineages that diverged in allopatry. The first lineage is limited to the oriental region whereas the second one is widespread, consisting of populations from New World, Afrotropical and Palearctic regions. Results from the AMOVA were consistent with this conclusion, indicating that great genetic

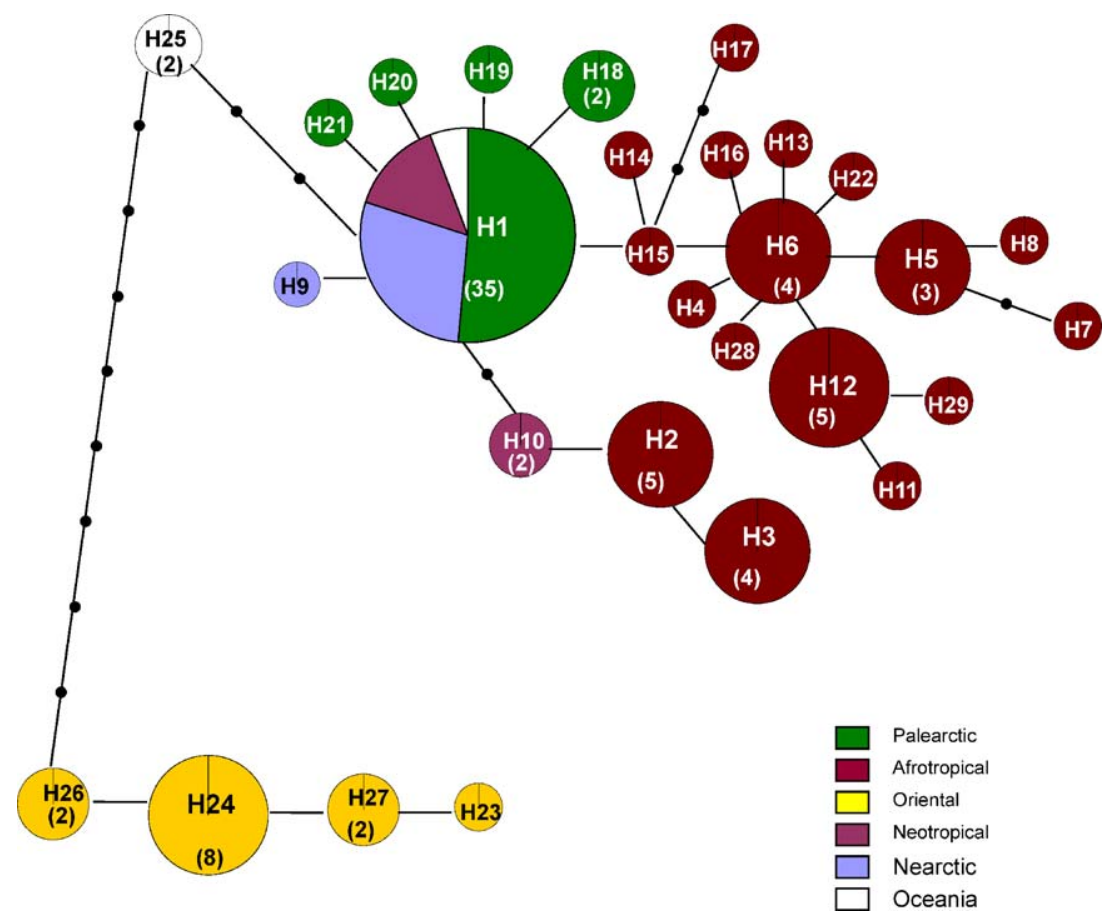

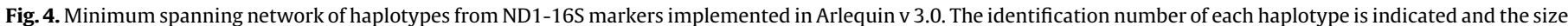
of circles is proportional to the number of sequences $(N)$ indicated in brackets $(N=1$ if not otherwise specified). Lines and full circles indicate mutational steps. 
Table 4

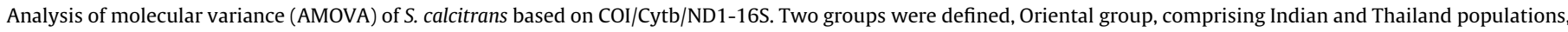
and group composed of remaining populations.

\begin{tabular}{|c|c|c|c|}
\hline Source of variation & Sum of squares & Variance components & Percentage of variation \\
\hline Among groups & $148.260 / 73.215 / 95.634$ & 7.527 Va/3.526Va/3.978 Va & $85.14 / 71.73 / 77.06$ \\
\hline Among populations within groups & $55.373 / 62.803 / 62.112$ & $0.644 \mathrm{Vb} / 0.751 \mathrm{Vb} / 0.712 \mathrm{Vb}$ & $7.29 / 15.28 / 13.79$ \\
\hline Within populations & $40.155 / 38.963 / 33.512$ & $0.669 \mathrm{Vc} / 0.638 \mathrm{Vc} / 0.472 \mathrm{Vc}$ & $5.57 / 12.99 / 9.14$ \\
\hline Fixation indices & \multirow{4}{*}{\multicolumn{2}{|c|}{$\begin{array}{l}0.490^{*} / 0.540^{*} / 0.604^{*} \\
0.907^{*} / 0.870^{*} / 0.908^{*} \\
0.851^{\mathrm{a}} / 0.717^{\mathrm{a}} / 0.770^{\mathrm{b}}\end{array}$}} & $P$-values \\
\hline Fsc & & & $<0.0001$ \\
\hline Fst & & & ${ }^{*}<0.0001$ \\
\hline Fct & & & ${ }^{\mathrm{a}} 0.007 ;{ }^{\mathrm{b}} 0.003$ \\
\hline
\end{tabular}

Fixation indices are Fsc genetic variation among populations within groups, Fst: genetic variation among all populations; Fct: genetic variation among groups.

Table 5

Results of neutrality tests (Tajima's D and Fu's Fs) and mismatch analyses for each mitochondrial marker for Afrotropical, Oriental and Palearctic populations.

\begin{tabular}{|c|c|c|c|c|c|}
\hline & & Tajima's $D$ & Fu's $F s$ & Raggedness index, $\operatorname{Rg}$ ( $P$ value) & Mismatch distribution, SSD ( $P$ values) \\
\hline $\mathrm{COI}$ & $\begin{array}{l}\text { Afrotropical } \\
\text { Oriental } \\
\text { Palearctic }\end{array}$ & $\begin{array}{l}-0.022^{\mathrm{NS}} \\
-0.515^{\mathrm{NS}} \\
-1.257^{\mathrm{NS}}\end{array}$ & $\begin{array}{l}-2.550^{\mathrm{NS}} \\
-1.866^{\mathrm{NS}} \\
-3.513^{*}\end{array}$ & $\begin{array}{l}0.057(P>0.05) \\
0.075(P>0.05) \\
0.161(P>0.05)\end{array}$ & $\begin{array}{l}0.003(0.430) \\
0.011(0.509) \\
0.022(0.194)\end{array}$ \\
\hline Cytb & $\begin{array}{l}\text { Afrotropical } \\
\text { Oriental } \\
\text { Palearctic }\end{array}$ & $\begin{array}{l}-0.789^{\mathrm{NS}} \\
-0.353^{\mathrm{NS}} \\
-0.925^{\mathrm{NS}}\end{array}$ & $\begin{array}{l}-2.787^{\mathrm{NS}} \\
-2.600^{*} \\
-2.703^{*}\end{array}$ & $\begin{array}{l}0.128(P>0.05) \\
0.080(P>0.05) \\
0.172(P>0.05)\end{array}$ & $\begin{array}{l}0.047(0.182) \\
0.013(0.402) \\
0.017(0.147)\end{array}$ \\
\hline ND1-16S & $\begin{array}{l}\text { Afrotropical } \\
\text { Oriental } \\
\text { Palearctic }\end{array}$ & $\begin{array}{l}-0.627^{\mathrm{NS}} \\
-0.478^{\mathrm{NS}} \\
-1.295^{\mathrm{NS}}\end{array}$ & $\begin{array}{l}-8.927^{*} \\
-0.940^{\mathrm{NS}} \\
-2.402^{*}\end{array}$ & $\begin{array}{l}0.031(P>0.05) \\
0.099(P>0.05) \\
0.173(P>0.05)\end{array}$ & $\begin{array}{l}0.009(0.275) \\
0.005(0.646) \\
0.226(0.000)\end{array}$ \\
\hline
\end{tabular}

NS: non significant values.

Significant at the 0.02 level.

variation in S. calcitrans was partitioned between the two groups. In contrast, a weak geographic structure was detected among populations within group.

Genetic differentiation in S. calcitrans was similar to pattern observed by Cummings et al. (2005) for the face flies, as they detect an enormous genetic differentiation between Kazakhstan and the more western regions of face fly's Old World range. They also notice the absence of an intermediary haplotype between flies from Kazakhstan and the other regions, suggesting the existence of a species complex.

Our data set shows the absence of haplotypes shared between the two groups, whereas a few shared haplotypes between Oriental region and the other regions was found by Marquez et al. (2007) based on their datasets. As well, they showed that Phylogenetic
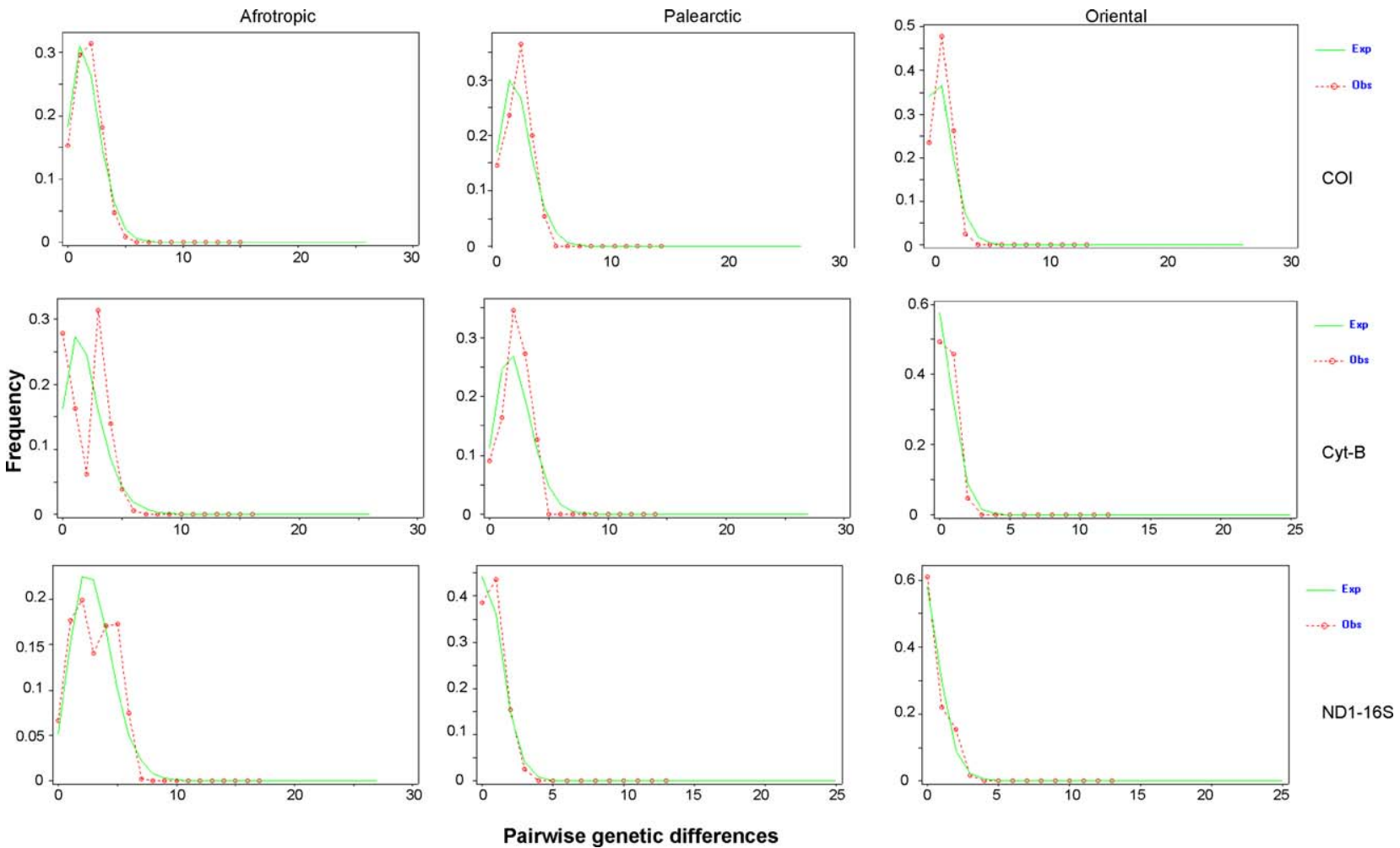

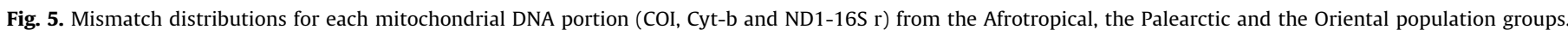


constructions demonstrate separate and genetically distant clusters between the Oriental and the Ethiopian regions. There is evidence for similar structure revealed by Cummings and Krafsur (2005), when they surveyed the mitochondrial COI diversity in worldwide house fly populations, and they observed a large number of private and singular haplotypes in the Indo-Malayan region, suggesting a considerable degree of genetic isolation.

The genetic distance suggest that the Oriental lineage has been isolated since 0.7-1 million years ago, corresponding approximately to mid Pleistocene. This period is characterized by repeated glaciation events, leading to drastic changes in the vegetation composition within different biomes. Avise et al. (1998) reported that among major intraspecific phylogroups identified in the mammals, herpetofauna and fishes, population divergence times fall within the Pleistocene.

During early Pleistocene, the Oriental region was the seat of many divergence events within Anopheles species, like A. sundicus complex or major species of the Neocellia series, suggesting that this region may have forest refugia during Pleistocene glacial periods (Dusfour et al., 2007; Morgan et al., 2009).

The strong S. calcitrans geographic structure, found between the Oriental populations and the remaining ones, could be explained by the isolation of the two groups in two different refugia: the Oriental refuge and the more Western refuge, during the Quaternary. This isolation was probably reinforced by the presence of biogeographic barriers (Himalayan Mountains, deserts...). Another reason to explain this isolation would be that $S$. calcitrans was already established in Eastern region for a long time and that they would have prevented the colonization of new invaders. Indeed, when populations have filled the space, it is generally much more difficult for those behind them to colonize, because they must disperse and reproduce logistically and not exponentially like the original scattered colonists (Hewitt, 2000). New sampling from additional Oriental populations would be interesting to localize the origin refuge in the Oriental region, from which dispersion was restricted and limited to the Oriental area.

Concerning the $S$. calcitrans group II, the Afrotropical region could contain the refuge zone. Unfortunately, genetic diversity could not be used here to attest the hypothesis, because of the disparity in the sampling size between regions. However, the Afrotropical region comprise 14 Stomoxys species, from which 12 are exclusively African, while the Palearctic and the New World regions are represented by only S. calcitrans. Marquez et al. (2007) reported that the sub-Saharan Africa was the most diverse. This assumes that within the second group, the Afrotropical populations would be the most ancestral and that they recolonized the other regions but less the Oriental one. AMOVA analyses showed little mitochondrial variation among populations within group II, attesting existence of gene flow. Stable fly is a strongly vagile species, with great flight capacities in order to search blood meal (Hogsette et al., 1987). Several studies, at the regional scale, indicate an important gene flow between populations of $S$. calcitrans despite the natural geographic barriers (Gilles et al., 2007; Dsouli-Aymes et al., 2009). Jones et al. (1991), Krafsur (1993) and Szalanski et al. (1996) showed a low differentiation among $S$. calcitrans populations in the United States.

According to Brues (1913), New World S. calcitrans populations have a Palearctic origin. In 2007, Marquez et al. confirmed this origin within the past 500 years. Our sampling does not enable to confirm or to disapprove the introduction age of S. calcitrans in New World; however we are in agreement with this dating and we find it suitable. More information about the pattern of S. calcitrans New World colonization, are offered by Marquez et al. (2007).

Our estimates for $S$. calcitrans expansion is approximately 12,000-7000 years ago, which predates enough the significant continental human expansions that are estimated to have begun between 70,000 and 50,000 years ago (Rogers and Harpending, 1992). Recent expansions in a variety of disease vectors, such as Anophelinae (Donnelly et al., 2001; Dusfour et al., 2007; Matthews et al., 2007), and black flies (Pramual et al., 2005) appear to be tied to human population expansions and/or to climate changes and to the rise of agriculture, providing significant new breeding sites and sources of bloodmeals (Coluzzi, 1982). Coluzzi (1982) proposed that because these species were dependent on humans for feeding and breeding sites, populations may have mirrored the growth in populations of humans and domestic animals during this period.

Within Stomoxys genus, S. calcitrans is the only species that extents its distribution. Mavoungou et al. (2008) reported that this species is abundant in areas where human presence was manifest, whereas other Stomoxys species are more related to forest or savanna area. Besides, investigations conducted by Gilles et al. (2005a,b) based on comparison between two Stomoxys species that co-occur in La Reunion, the cosmopolitan S. calcitrans and the tropical Stomoxys niger niger Macquart, show that $S$. calcitrans breeds continuously whereas $S$. niger evolved a strategy of survival without any reproduction during the tropical winter, and that $S$. calcitrans is better adapted than $S$. niger, in terms of adult production from eggs. Thus, $S$. calcitrans is notable by its reproductive success.

In East Africa, where human density is high, Ayala and Coluzzi (2005) showed that A. arabiensis gradually became anthropophilitic and succeeds to adapt human environments. In 2004, Lachaise and Silvain suggested that the wild - to domestic behavior shift in D. melanogaster may date back to 18,000 years before present. The shift to domestic habit is an evolutionary response for species that are unable to adapt readily to changing natural environments, since $D$. melanogaster is unable to delay reproduction whenever this would be necessary (Lachaise and Silvain, 2004). The reproductive success of $S$. calcitrans could explain its worldwide distribution and its close relation to human activities.

Throughout this study, we were able to identify two S. calcitrans lineages. The first one is wholly Oriental, whereas the second is consisting of the remaining populations. The strong isolation between them may go back to the end of early Pleistocene. Human expansion and his new lifestyle (changes in agricultural practices and increase in blood supply) seems to be key for the establishment of $S$. calcitrans expansion process since it is a quintessence synanthropic fly.

\section{Acknowledgments}

We thank Jacques-François Mavoungou (Gabon), Roundthip Masmeatathip (Thailand), Alekaw Sinshaw Tegegne (Ethiopia), Dr. Nicolas Barre (New-Caledonia), Dr. Douglas D. Colwell (samples from Canada), Dr. J. Depaquit (Montenegro), Dr. J. Gilles (Madagascar, La Réunion, Mayotte), Dr. P. Grimaud (Uganda), Dr. J. Bouyer (Burkina Faso) and Dr. D. Cuisance (Cameroun) for providing some Stomoxys samples. We extend our thanks to Gwenaëlle Mondor (CBGP) for technical assistance. This study was partially supported by Agence Nationale de la Recherche through the ANR-Biodiv grant IFORA (Iles Forestières Africaines) and by the CNS-Genoscope "Barcoding insects for identification" sequencing grant to Michel Veuille.

\section{References}

Avise, J.C., 2000. Phylogeography. In: The History and Formation of Species, Havard University Press, Cambridge, MA, $464 \mathrm{p}$.

Avise, J.C., Walker, D., Johns, G.C., 1998. Speciation durations and Pleistocene effects on vertebrate phylogeography. Proc. R. Soc. Lond. B 265, 1707-1712.

Ayala, F.J., Coluzzi, M., 2005. Chromosome speciation: humans, Drosophila, and mosquitoes. PNAS 102, 6535-6542.

Bailey, D.L., Whitfield, T.L., Smittle, B.J., 1979. Flight and dispersal of the stable fly. J. Econ. Entomol. 66, 410-411. 
Batista, Z.R., Leite, R.C., Oliveira, P.R., Lopes, C.M.L., Borges, L.M.F., 2005. Populational dynamics of Stomoxys calcitrans (Linneaus) (Diptera: Muscidae) in three biocenosis, Minas Gerais. Braz. Vet. Parasitol. 130, 343-346.

Brower, A.V., 1994. Rapid morphological radiation and convergence among races of the butterfly Heliconius erato inferred from patterns of mitochondrial DNA evolution. PNAS 91, 6491-6495.

Brues, C.T., 1913. The geographical distribution of the stable fly S. calcitrans. J. Econ. Entomol. 6, 459-477.

Bruce, W.N., Decker, G.C., 1958. The relationship of stable fly to milk production in dairy cattle. J. Econ. Entomol. 51 (3), 269-275.

Campbell, J.B., Skoda, S.R., Berkerile, D.R., Boxler, D.J., Thomas, G.D., Adams, D.C., Davis, R., 2001. Effects of stable flies (Diptera: Muscidae) on weight gains of grazing yearling cattle. J. Econ. Entomol. 94, 780-783.

Campbell, J.B., White, R.G., Wright, J.E., Crookshank, R., Clanton, D.C., 1977. Effects of stable flies (Diptera: Muscidae) on weight gains and feed-efficiency of calves on growing and finishing rations. J. Econ. Entomol. 70, 592-594.

Coluzzi, M., 1982. Spatial distribution of chromosomal inversions and speciation in the Anopheline mosquitoes. Prog. Clin. Biol. Res. 96, 143-153.

Cummings, M.A., Moon, R.D., Krafsur, E.S., 2005. North American face flies old World origins: mitochondrial evidence. Med. Vet. Entomol. 19, 48-52.

Cummings, M.A., Krafsur, E.S., 2005. Spatial diversity in mitochondrial cytochrome c oxidase in house flies. Med. Vet. Entomol. 19, 53-59.

D’Amico, F., Gouteux, J.P., Le Gall, F., Cuisance, D., 1996. Are stable fly (Diptera: Stomoxyinae) vectors of Trypanosoma vivax in the Central African Republic. Vet. Res. 27, 161-170.

Donnelly, M.J., Licht, M.C., Lehmann, T., 2001. Evidence for recent population expansion in the evolutionary history of the malaria vectors Anopheles arabiensis and Anopheles gambiae. Mol. Biol. Evol. 18, 1353-1364.

Dsouli-Aymes, N., Mavoungou, J.F., De Stordeur, E., Duvallet, G., 2009. Landscape, population structure and genetic diversity of Stomoxys calcitrans. Parasite 16, 37-41.

Dusfour, I., Michaux, J.R., Harbach, R.E., Manguin, S., 2007. Speciation and phylogeography of the Southeast Asian Anopheles sundaicus complex. Infect. Genet. Evol. 7, 484-493.

Eddy, G.H., Rooth, A.R., Plapp, F.W., 1962. Studies on the flight habitats of some marked insects. J. Econ. Entomol. 55, 603.

Eesa, N.M., El-Sibae, M.M., 1993. Population dynamics of some synanthropic fly species in different habitats in Buraydah, Saudi Arabia. J. Egypt. Soc. Parasitol. 23, 133-140.

Excoffier, L., Laval, G., Schneider, S., 2005. Arlequin ver. 3.0: an integrated software package for population genetics data analysis. Evol. Bioinform. Online 1, 47-50.

Foil, L.D., Gorham, J.R., 2000. Mechanical transmission of disease agents by arthropods. In: Eldridge, B.F., Edman, J.D. (Eds.), Med. Entomol.: A Textbook on Public Health and Veterinary Problems Caused by Arthropods. Kluwer Academic Publishers, pp. 461-514.

Foil, L.D., Hogsette, J.A., 1994. Biology and control of tabanids, stable flies and horn flies. Rev. Sci. Tech. 13, 1125-1158.

Foil, L.D., Meek, C.L., Adams, W.V., Issel, C.J., 1983. Mechanical transmission of equine infectious anemia virus by deer flies (Chrysops flavidus) and stable flies (Stomoxys calcitrans). Am. J. Vet. Res. 44, 155-156.

Fu, Y.X., 1997. Statistical tests of neutrality of mutations against population growth, hitchhiking and backgroud selection. Genetics 147, 915-925.

Freitas, T.R., Romero, C.H., 1991. Experimental transmission of bovine leucosis virus by leucocytes recovered from the stable fly Stomoxys calcitrans (L.). Braz. J. Med. Biol. Res. 24, 101-123.

Gilles, J., David, J.F., Duvallet, G., 2005a. Temperature effects on development and survival of two stable flies, Stomoxys calcitrans and Stomoxys niger (Diptera: Muscidae), in La Réunion Island. J. Med. Entomol. 42, 260-265.

Gilles, J., David, J.F., Duvallet, G., 2005b. Effects of temperature on the rate of increase of two stable flies from La Réunion island, Stomoxys calcitrans and Stomoxys niger niger (Diptera: Muscidae). J. Med. Entomol. 42, 959-965.

Gilles, J., Litrico, I., Tillard, E., Duvallet, G., 2007. Genetic structure and gene flow along an altitudinal gradient among two Stomoxyine species (Diptera: Muscidae) on La Réunion Island. J. Med. Entomol. 44, 433-439.

Guindon, S., Gascuel, O., 2003. A simple, fast and accurate algorithm to estimate large phylogenies by maximum likelihood. Syst. Biol. 52, 696-704.

Hall, T.A., 1999. BioEdit: a user-friendly biological sequence alignment editor and analysis program for Windows 95/98/NT. Nucleic Acids Symp. Ser. 41, 95-98.

Hasegawa, M., Kishino, H., Yano, T., 1985. Dating of the human-ape splitting by a molecular clock of mitochondrial DNA. J. Mol. Evol. 22, 160-174.

Hewitt, G.M., 2000. The genetic legacy of the quaternary ice ages. Nature 405, $907-$ 913.

Hogsette, J.A., Ruff, J.P., Jones, C.J., 1987. Stable fly biology and control in the northwest Florida. J. Agric. Entomol. 4, 1-11.

Jones, C.J., Hogsette, J.A., Patterson, R.B., Milne, D.E., Propp, D.G., Milio, J.F., Rickard, L.R., Ruff, J.P., 1991. Origin of the stable flies (Diptera: Muscidae) on west Florida beaches: electrophoretic analysis of stable fly dispersal. J. Med. Entomol. 28, 787-795.

Krafsur, E.S., 1993. Allozyme variation in stable flies (Diptera: Muscidae). Biochem. Genet. 31, 231-239.

Lachaise, D., Silvain, J.F., 2004. How two Afrotropical endemics made two cosmopolitan human commensals: the Drosophila melenogaster-D.simulans paleogeographic riddle. Genetica 120, 17-39.

Laveissière, C., Grébaut, P., 1990. The trapping tsetse flies (Diptera: Glossinidae) Improvement of a model: the Vavoua trap. Trop. Med. Parasitol. 41, 185-192.

Lysyk, T.J., 1998. Relationships between temperature and life-history parameters of Stomoxys calcitrans (Diptera: Muscidae). J. Med. Entomol. 35, 107-119.

Marquez, J.G., Cummings, M.A., Krafsur, E.S., 2007. Phylogeography of stable fly (Diptera: Muscidae) estimated by diversity at ribosomal 165 and Cytochrome Oxidase I mitochondrial genes. J. Med. Entomol. 44, 998-1008.

Matthews, S., Meehan, D.L.J., Onyabe, D.Y., Vineis, J., Nock, I., Ndams, I., Conn, J.E., 2007. Evidence for late Pleistocene population expansion of the malarial mosquitoes, Anopheles arabiensis and Anopheles gambiae in Nigeria. Med. Vet. Entomol. 21, 358-369.

Mavoungou, J.F., Jay-Robert, P., Gilles, J., Atsame, E.A., Duvallet, G., 2008. Ecology of Stomoxys flies (Diptera: Muscidae) in Gabon, First survey in different ecological areas. Parasite 151, 27-34.

Miller, R.W., Pickens, L.G., Morgan, N.O., Thimijan, R.W., Wilson, R.L., 1973. Effect of stable flies on feed intake and milk production of dairy cows. J. Econ. Entomol. $66,711-713$.

Morgan, K., O’Loughlin, S.M., Mun-Yik, F., Linton, Y.M., Somboon, P., Min, S., Than Htun, P., Nambanya, S., Weerasinghe, I., Sochantha, T., Prakash, A., Walton, K. 2009. Molecular phylogenetics and biogeography of the Neocellia Series of Anopheles mosquitoes in the oriental region. Mol. Phylogenet. Evol. 52, 588601.

Posada, D., Crandall, K.A., 1998. MODELTEST: testing the model of DNA substitution Bioinformatics 14, 817-818.

Pramual, P., Kuvangkadilok, C., Baimai, V., Walton, C., 2005. Phylogeography of the Black fly Simulium tani (Diptera: Simuliidae) from Thailand as inferred from mtDNA sequences. Mol. Ecol. 14, 3989-4001.

Rogers, A.R., Harpending, H., 1992. Population growth makes waves in the distribution of pairwise genetic differences. Mol. Biol. Evol. 9, 552-569.

Rozas, J., Sanchez-DelBarrio, J.C., Messeguer, X., et al., 2003. DnaSP, DNA polymorphism analyses by the coalescent and other methods. Bioinformatics 19, 24962497.

Sharpe, R.G., Harbach, R.E., Butlin, R.K., 2000. Molecular variation and phylogeny of members of the Minimus group of Anopheles subgenus Cellia (Diptera: Culicidae). Syst. Entomol. 25, 263-272.

Simon, C., Buckley, T.R., Frati, F., Stewart, J.B., Beckenbach, A.T., 2006. Incorporating molecular evolution into phylogenetic analysis, and a new compilation of conserved polymerase chain reaction primers for animal mitochondrial DNA. Supplemental material. Ann. Rev. Ecol. Evol. Syst. 37, 545-579.

Simon, C., Frati, F., Beckenbach, A., Crespi, B., Lui, H., Flook, P., 1994. Evolution, weighting and phylogenetic unity of mitochondrial gene sequences and a compilation of conserved polymerase chain reaction primers. Ann. Entomol. Soc. 87, 651-701.

Swofford, D.L., 2002. PAUP*: Phylogenetic Analysis Using Parsimony (*and Other Methods). Sinauer Associates, Sunderland MA.

Szalanski, A.L., Taylor, D.B., Peterson, R.D., 1996. Population genetics and gene variation of stable fly populations (Diptera: Muscidae) in Nebraska. J. Med. Entomol. 33, 413-420.

Tamura, K., Nei, M., 1993. Estimation of the number of nucleotide substitutions in the control region of mitochondrial DNA in humans and chimpanzees. Mol. Biol. Evol. 10, 512-526.

Tamura, K., Nei, M., Kumar, S., 2004. Prospects for inferring very large phylogenies by using the neighbor-joining method. Proc. Natl. Acad. Sci. U.S.A. 101, 11030 11035 .

Tamura, K., Dudley, J., Nei, M., Kumar, S., 2007. MEGA4: molecular evolutionary genetics analysis (MEGA) software version 4.0. Mol. Biol. Evol. 24, 15961599.

Tajima, F., 1989. Statistical method for testing the neutral mutation hypothesis by DNA polymorphism. Genetics 123, 585-595.

Thompson, J.D., Higgins, D.G., Gibson, T.J., 1994. CLUSTAL W: improving the sensitivity of progressive multiple sequence alignment through sequence weighting, position specific gap penalties and weight matrix choice. Nucleic Acids Res. 22, 4673-4680.

Wieman, G.A., Campbell, J.B., Deshazer, J.A., Berry, I.L., 1992. Effects of stable flies (Diptera, Muscidae) and heat-stress on weight-gain and feed-efficiency of feeder cattle. J. Econ. Entomol. 85, 1835-1842.

Zumpt, F., 1973. The Stomoxyinae biting flies of the world. In: Taxonomy, Biology, Economic Importance and Control Measures, Gustav Fischer Verlag, Stuttgart, $175 \mathrm{p}$. 